\title{
SUR LE PHLEBOTOMUS CHINENSIS
}

\author{
Par Virgil NITZULESCU
}

Nous avons trouvé, dans la collection du Laboratoire de Parasitologie de la Faculté de médecine de Paris, un lot de Phlebotomus chinensis provenant de Pékin, d'où ils avaient été envoyés par le $\mathrm{D}^{\mathrm{r}}$ Young au Prof. Brumpt (1) en 1927. Le lot comprenait 11 individus, 8 mâles et 3 femelles, montés à l'érénol en préparations microscopiques.

Tous ces phlébotomes présentaient des caractères communs. Ils appartenaient manifestement à la même race. Il nous a semblé intéressant de donner la description de cette race, car il n'existe à notre connaissance, à l'heure actuelle, aucune description détaillée du Phlebotomus chinensis, provenant de Chine, donc bien authentique. Les descriptions que nous avons données pour les formes européennes doivent nécessairement être comparées avec celle-là. Or la description de Newstead (1916) est extrêmement sommaire. Celle de Patton (1926), bien plus détaillée, reste inutilisable, parce qu'on n'y trouve rien sur les éléments principaux de la diagnose : filaments génitaux et tubercule de l'organe intromittent des mâles, spermathèques des femelles. La description plus récente d'Adler (1929), tout en apportant des renseignements précieux, reste elle aussi trop sommaire pour que nous puissions nous faire d'après elle une idée précise de cette espèce. Sinton (1928) ne s'occupe que de la spermathèque des femelles et très peu des filaments génitaux du mâle. Du texte de Sinton, il ressort que les filaments génitaux du $P$. chinensis sont très longs et c'est justement ce point qui nous faisait hésiter dans la détermination que nous venons de faire pour les exemplaires de Skoplje. Par contre, dans la figure donnée plus récemment encore par Patton (1929), les filaments génitaux du $P$. chinensis sont représentés comme relativement courts (à peine 4 fois et demie la longueur de la pompe) et la pompe elle-même est figurée comme descendant très bas.

Nous ne devons pas oublier que $P$. chinensis est vecteur du kala-azar en Asie. Sa présence en Europe est donc assez inquié-

(1) Nous remercions très vivement M. le Prof. Brumpt qui a bien voulu nous communiquer ce lot de phlébotomes.

Annales de Parasitologie, T. VIII, $\mathrm{N}^{\mathrm{os}} 3-4,-1^{\text {er }}$ juillet 1930 , p. 362-375. 
tante. C'est un motif de plus pour étudier dans leurs moindres détails toutes les formes de $P$. chinensis d'Asie, afin de contrôler si les formes d'Europe s'y superposent exactement et de voir si l'on pourrait trouver des données morphologiques représentant des corrélations de caractères qui permettraient de créer des subdivisions dans le sein de cette espèce, subdivisions dont la biologie et la capacité de transmettre des germes pourraient être tout autres.

Pour le moment, un tel travail serait prématuré. Il faut que l'on récolte d'abord beaucoup de matériel de comparaison en Europe et en Asie pour pouvoir le tenter.

Nous devons ajouter qu'à Bucarest, alors que nous avons commencé ces recherches (Nitzulescu, 1929), nous avons vainement essayé de nous procurer le matériel asiatique dont nous avions grandement besoin pour les identifications. Il nous a été bien difficile de la sorte de faire en toute certitude la diagnose des formes balkaniques. Nous croyons de notre devoir d'éviter aux autres chercheurs, qui seraient obligés de travailler dans des conditions similaires, une peine inutile en leur présentant sous forme de chiffres une partie de ce matériel.

La " phlébotomométrie " a été et reste encore sujette à beaucoup de reproches (Magnitski et Gutzewitsch, 1929). Evidemment, les chiffres obtenus chez des individus isolés n'ont qu'une valeur relative. Mais, si l'on extrait les moyennes mathématiques des nombreuses mensurations effectuées dans un même lot, on obtient une série de chiffres qui permettent d'établir des rapports correspondant à des corrélations des divers organes. Ces chiffres, étant des moyennes, éliminent forcément les petits écarts individuels. Nous nous sommes donc servi de cette méthode, sauf dans les cas où malheureusement nous n'avons pas pu le faire, comme pour les articles antennaires des femelles. En effet, des trois femelles que nous avons pu étudier, une seule possédait une de ses antennes. De même, quand il s'agissait de rapports trop variables, nous l'avons indiqué et nous avons donné à part les chiffres de chaque individu. Tels sont les rapports $\frac{\alpha}{\beta}$ des ailes ou les formules des palpes. Par contre, là où nous n'avons donné que des moyennes sans autre indication, les rapports que nous avons obtenus en comparant les divers chiffres entre eux correspondaient assez étroitement aux mêmes rapports pris dans chaque individu isolé. 


\section{Description Des MaLes}

\section{Dimensions}

Nous avons mesuré 5 mâles dont la taille était respectivement : $2.490,2.650,2.560,2.610$ et $2.645 \mu$.

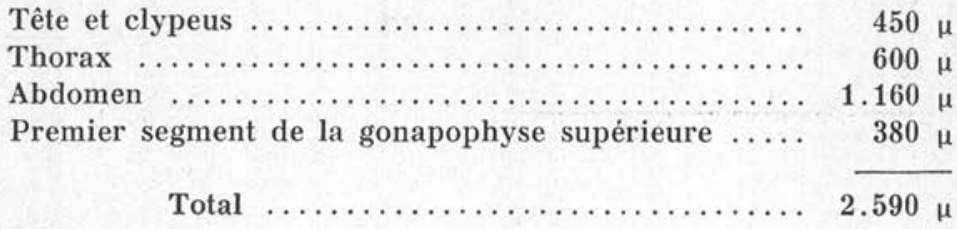

\section{Antennes}

\begin{tabular}{|c|c|c|}
\hline Segm. I . . . . . . & $70 \mu$ & \\
\hline Segm. II $\ldots \ldots \ldots \ldots \ldots \ldots$ & $65 \mu$ & \\
\hline Segm. III $\ldots \ldots \ldots \ldots \ldots$ & $454 \mu$ & \\
\hline Segm. IV $\ldots \ldots \ldots \ldots \ldots$. & $198 \mu$ & \\
\hline Segm. $v \ldots \ldots \ldots \ldots \ldots$ & $201 \mu$ & III $>$ IV + V \\
\hline Segm. VI $\ldots \ldots \ldots \ldots \ldots$. & $193 \mu$ & $\mathrm{III}<\mathrm{IV}+\mathrm{V}+\mathrm{VI}$ \\
\hline Segm. VII $\ldots \ldots \ldots \ldots \ldots$ & $177 \mu$ & III $>$ XII.....XVI \\
\hline Segm. VIII $\ldots \ldots \ldots \ldots \ldots$ & $168 \mu$ & IV + V $=$ XII - XVI. - Dans un \\
\hline Segm. IX $\ldots \ldots \ldots \ldots \ldots$ & $146 \mu$ & cas nous avons trouvé : \\
\hline Segm. $X \ldots \ldots \ldots \ldots$ & $130 \mu$ & $\mathrm{IV}+\mathrm{V}=380$ \\
\hline Segm. XI $\ldots \ldots \ldots \ldots \ldots$ & $117 \mu$ & $\mathrm{XII}-\mathrm{XVI}=420$. \\
\hline Segm. XII $\ldots \ldots \ldots \ldots \ldots$ & $105 \mu$ & $\mathrm{IV}+\mathrm{V}+\mathrm{VI}>\mathrm{XII}-\mathrm{XVI}$ \\
\hline Segm. XIII $\ldots \ldots \ldots \ldots \ldots$ & $81 \mu$ & \\
\hline Segm. XIV $\ldots \ldots \ldots \ldots \ldots$ & $66 \mu$ & \\
\hline Segm. XV $\ldots \ldots \ldots \ldots \ldots$ & $61 \mu$ & \\
\hline Segm. XVI $\ldots \ldots \ldots \ldots \ldots$ & $71 \mu$ & \\
\hline
\end{tabular}

Il y a deux épines géniculées pour chaque article antennaire, du $3^{e}$ jusqu'au $15^{\circ}$. La formule antennaire est donc $\frac{2}{\text { III-XV }}$. Sur le troisième segment, les épines s'insèrent dans la moitié antérieure, sur les autres dans la moitié postérieure. Plus on avance vers l'extrémité de l'antenne, plus les épines sont petites et difficilement visibles.

\section{Palpes}

Les palpes se montrent extrêmement variables. Dans un exemplaire nous avons trouvé :

Segm. I ............ $45 \mu$

Segm. II $\ldots \ldots \ldots \ldots \ldots \ldots .147 \mu$

Segm. III $\ldots \ldots \ldots \ldots \ldots \ldots .180 \mu$

Segm. IV ............ $145 \mu$

Segm. V ............ $250 \mu$
Done : II $=$ IV
$\begin{array}{ll}\text { II }<\text { III } & \text { V } \\ \text { II }+ \text { III }>\text { V } & \text { IV }\end{array}$
III + IV $>$ V

Formule palpale : $1(2,4), 3,5$. 
Dans un autre nous avons trouvé :
Segm. I . . . . . . . 50 .
Donc : II $>$ IV
Segm. II ............. $168 \mu$
Segm. III $\ldots \ldots \ldots \ldots \ldots \ldots .205 \mu$
Segm. IV ........... $135 \mu$
$\begin{array}{ll}\text { II }<\text { III } & \text { V }=3\end{array}$
Segm. V
$405 \mu$

Dans un autre exemplaire le rapport $\frac{\mathrm{V}}{\mathrm{IV}}=2,4$ et la formule est : $1(2,4), 3,5$.

Il est à remarquer que dans ce petit lot d'insectes dont la plupart des mensurations présentaient des rapports étonnamment concordants, les palpes étaient l'organe le plus variable.

\section{Pattes}

Moyennes prises sur trois des individus.

Première paire de pattes :

Fémur

Tibia

Tarse

Tarse 2

Tarses 3-5
940

$1.160 \mu$

720 is

$332 \mu$

$470 \mu$ $\frac{\text { Tarse } 1}{\text { Tarse } 2}=2,17$

$$
\begin{aligned}
& \text { Total } \ldots \ldots \ldots \ldots \quad 3.622 \mu \\
& \frac{\text { Patte } 1 \text { (sans coxa ni trochanter) }}{\text { Taille de l'insecte }}=1,4 .
\end{aligned}
$$

Deuxième paire de pattes :

\begin{tabular}{|c|c|c|}
\hline Fémur $\ldots$ & $1.010 \mu$ & \\
\hline Tibia $\ldots \ldots \ldots \ldots \ldots$ & $1.756 \mu$ & \\
\hline Tarse $1 \ldots \ldots \ldots \ldots$ & $955 \mu$ & \\
\hline Tarse $2 \ldots \ldots \ldots \ldots \ldots$ & $397 \mu$ & $\frac{\text { Tarse } 1}{\text { Tarse } 2}=2,40$. \\
\hline Tarses $3-5 \ldots \ldots \ldots \ldots$ & $530 \mu$ & \\
\hline Total & 4.648 । & \\
\hline
\end{tabular}

$$
\begin{aligned}
& \text { Fémur .................... } 870 \mu \\
& \text { Tibia ............. } 1.406 \mu \\
& \text { Tarse } 1 \ldots \ldots \ldots \ldots \ldots .601 \mu \\
& \text { Tarse } 2 \ldots \ldots \ldots \ldots \ldots . \quad 368 \mu \\
& \text { Tarses } 3-5 \ldots \ldots \ldots \ldots \ldots . \quad 478 \mu \quad \overline{\text { Tarse } 2}=2,17 \text {. } \\
& \text { Total ........ } 3.923 \mu \\
& \frac{\text { Patte } 2 \text { (sans coxa ni trochanter) }}{\text { Taille de l'insecte }}=1,5
\end{aligned}
$$

Troisième paire de pattes: 


\section{$\frac{\text { Patte } 3 \text { (sans coxa ni trochanter) }}{\text { Taille de l'insecte }}=1,8$.}

\section{Aile}

Le bord postérieur de l'aile est plus arqué que le bord antérieur. Le maximum de largeur est en arrière de $\mathrm{Cu}^{2}$. La fourche de la deuxième nervure longitudinale est presque au même niveau que la fourche de la quatrième (fig. 1).

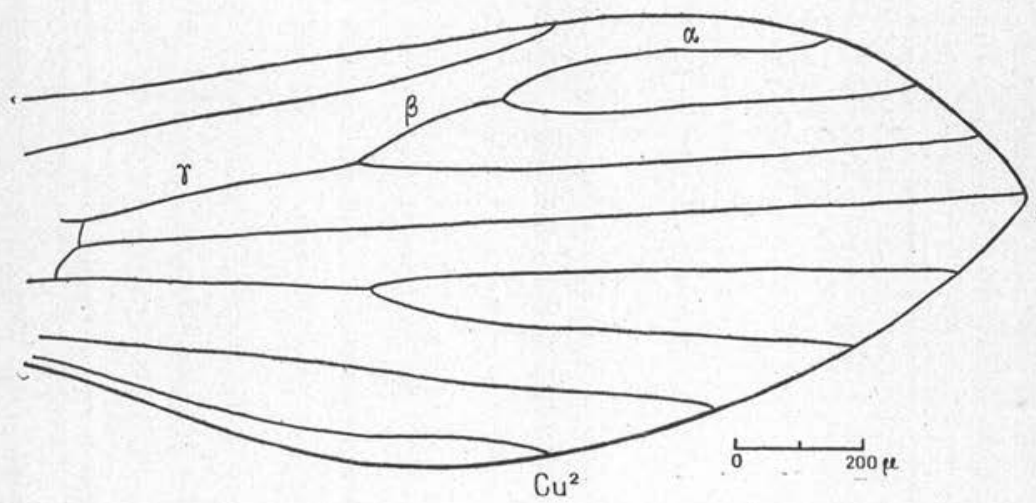

FIg. 1. - Phlebotomus chinensis. Aile d'un mâle. La plus grande largeur est atteinte en arrière de $\mathrm{Cu}^{2}$.

Longueur de l'aile .......... 2.315 $\mu$

Largeur maxima ............ $650 \mu$ (moyenne des 5 individus)

$$
\begin{aligned}
& \frac{\text { Longueur de l'aile }}{\text { Largeur de l'aile }}=3,56 \text {. } \\
& \frac{\text { Taille de l'insecte }}{\text { Longueur de l'aile }}=1,1 \text {. }
\end{aligned}
$$

$\alpha$ et $\gamma$ sensiblement égales, en moyenne $475 \mu$.

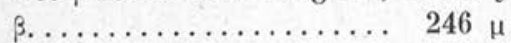

$\delta \ldots \ldots \ldots \ldots \ldots \ldots \ldots \ldots \ldots \ldots$

Voici quelques rapports $\frac{\alpha}{\beta}$

$$
\frac{\alpha=450}{\beta=250}=1,8 \frac{\alpha=475}{\beta=246}=1.9 \frac{\alpha=510}{\beta=250}=2,04
$$

2,04 est toutefois le plus élevé des rapports que nous avons trouvés. 


\section{Armature génitale}

Le deuxième segment de la gonapophyse supérieure présente cinq épines, plus courtes que le segment qui les porte. Leurs longueurs respectives sont inégales. Une des deux internes paraît un peu plus mince que les autres (fig. 2).

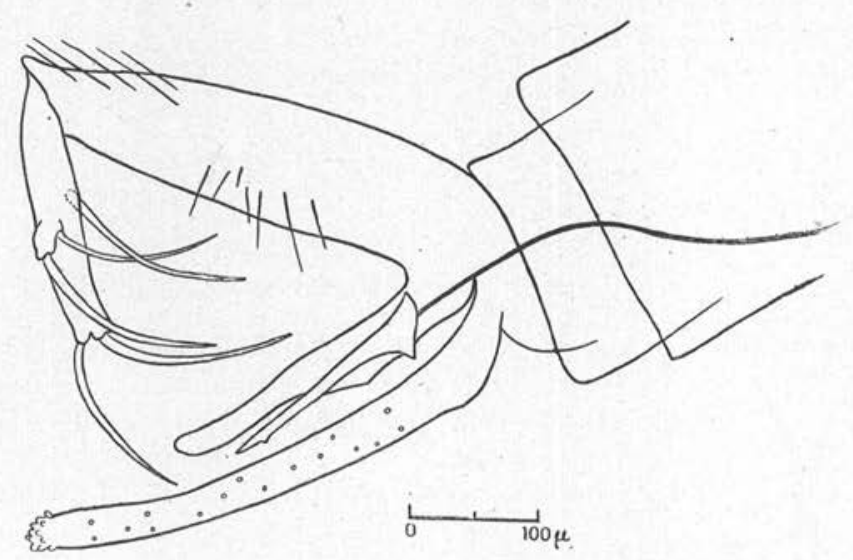

Fıg. 2. - Phlebotomus chinensis. Armature génitale du mâle.

La touffe de poils du premier segment de la gonapophyse supérieure, qui était très marquée sur les exemplaires de Bucarest et assez marquée

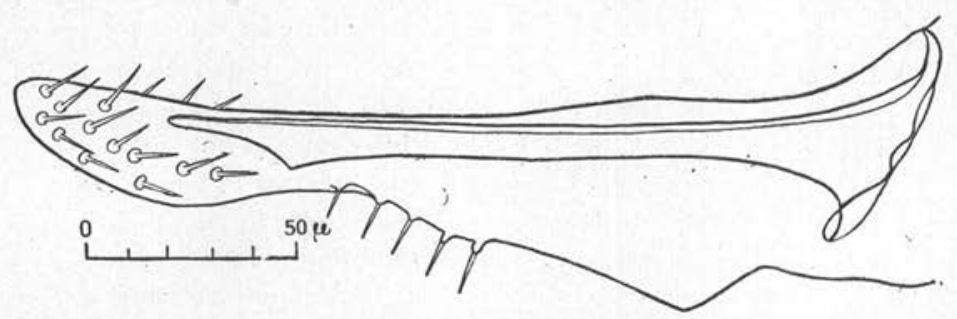

Fıg. 3. - Phlebolomus chinensis. Appendice intermédiaire et organe intromittent.

pour ceux de Skoplje, est par contre très réduite dans cette race. L'organe intromittent présente un tubercule inférieur. Ce tubercule mérite à peine le nom de subapical, car, comme on le voit sur la figure 3 et comme d'ailleurs Adler (1929) l'a déjà représenté, il est bien plus loin de l'extrémité de l'organe que dans les formes que nous avons décrites en Europe. Sur la même figure 3, on verra le rapport entre l'extrémité antérieure de l'organe intromittent et l'extrémité antérieure de l'appendice 
intermédiaire, rapport que nous avons trouvé toujours constant. Voici des chiffres moyens des différentes parties de l'armature génitale :

Gonapophyse supérieure $\left\{\begin{array}{l}\text { Segm. I } \ldots \ldots \ldots \ldots \ldots \ldots \ldots \ldots \ldots \\ \text { Segm. } 380 \mu\end{array}\right.$

$\left\{\begin{array}{l}\text { Segm. II } \ldots \ldots \ldots \ldots \ldots \ldots \ldots \ldots \ldots \\ 215 \mu\end{array}\right.$

Epines

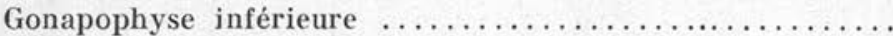

$416 \mu$

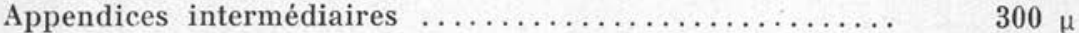

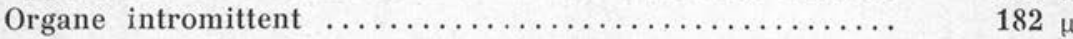

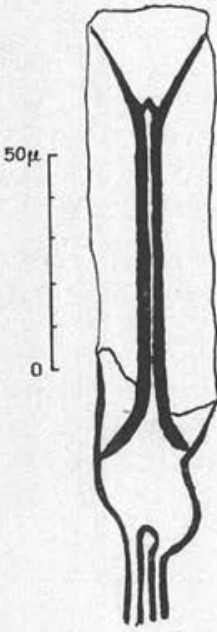

Fig. 4. - Phlebotomus chinensis.

Pompe génitale.

Segm. 1 de la gon. sup. $=1,7$.

Segm. 2 de la gon. sup.

Segm. 1 de la gon. sup. $=1,14$.

Gonapophyse inf.

\section{Pompe et filaments génitaux}

La pompe est largement évasée dans sa partie supérieure (fig. 4). Les filaments génitaux sont sensiblement comparables en longueur à ceux du $P$. longiductus décrit yar Perfiliew (1928) ou à ceux que nous avons rencontrés en Yougoslavie. En effet, le rapport filament-pompe ne dépasse jamais 7. Il s'abaisse même jusqu'à 5,5 dans un exemplaire. Donc, même pour les phlébotomes de Chine, les filaments génitaux ne sont pas nécessairement excessivement longs. Ils ont tendance, d'autre part, à sortir sur une grande longueur des organes intromittents en attirant la pompe vers les derniers segments abdominaux. Chez un exemplaire, la pompe se trouvait dans le tout dernier segment.

C'est à César Pinto (1926) que nous devons le premier essai de caractérisation de certains phlébotomes par la forme et par la position de la pompe et par la longueur des filaments génitaux. C'est un caractère facilement visible et assurément très utile, mais les limites dans lesquelles il peut os̄ciller sont, du moins chez $P$. chinensis, fort larges.

\section{DESCRIPTION DES FEMELLES}

\section{Dimensions}

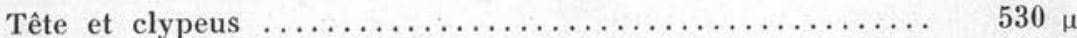

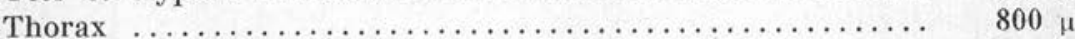

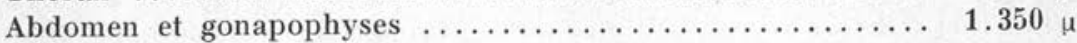




\section{Antennes}

Une seule antenne a été mesurée :

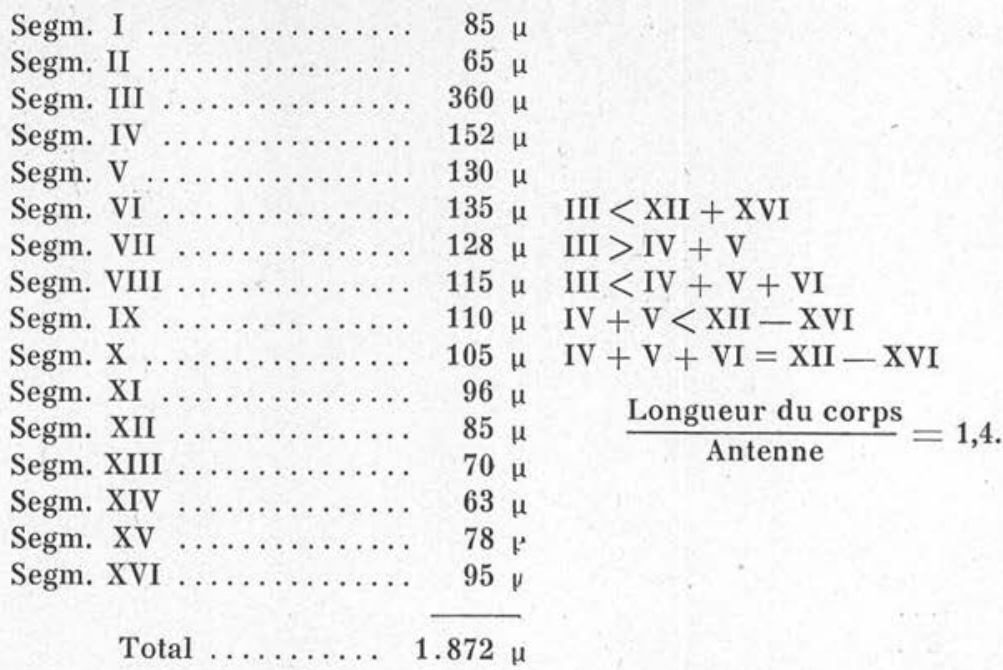

Les épines géniculées sont disposées comme chez les mâles : $\frac{2}{\mathrm{XII}-\mathrm{X}} \overline{\mathrm{V}}$. Elles sont bien plus longues que chez eux, surtout celles qui sont vers l'extrémité distale. Elles n'atteignent jamais l'extrémité antérieure de l'article sur lequel elles sont insérées.

\section{Palpes}

Une seule femelle laissait bien mesurer ses palpes. En voici les dimensions :

Segm. I ............ $65 \mu$

$\begin{array}{llll}\text { Segm. II } \ldots \ldots \ldots \ldots \ldots \ldots & 225 \mu & \text { II }+ \text { III }<\text { V } \\ \text { Segm. III } \ldots \ldots \ldots \ldots \ldots \ldots & 240 \mu & \text { III }+ \text { IV }<\text { V }\end{array} \quad \frac{\text { V }}{\text { IV }}=1,6$

Segm. IV $\ldots \ldots \ldots \ldots \ldots \ldots .190 \mu$ Formule palpale : 1, 4, 2, 3, 5 .

Segm. V ............ $305 \mu$

\section{Pattes}

Première paire de pattes : 


$$
\frac{\text { Patte } 1 \text { (sans coxa ni trochanter) }}{\text { Longueur du corps }}=1,3 .
$$

Deuxième paire de pattes :

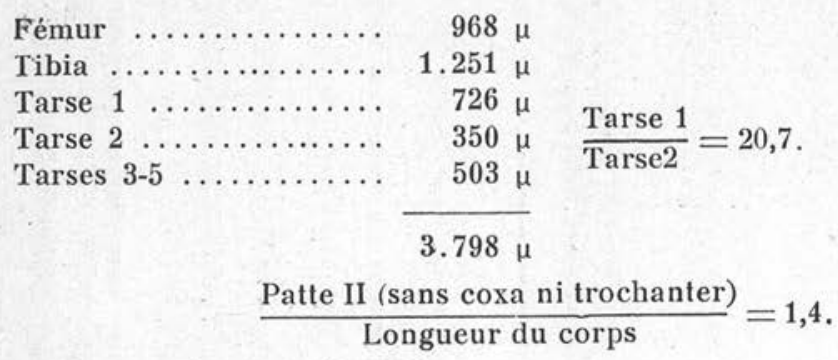

Troisième paire de pattes :

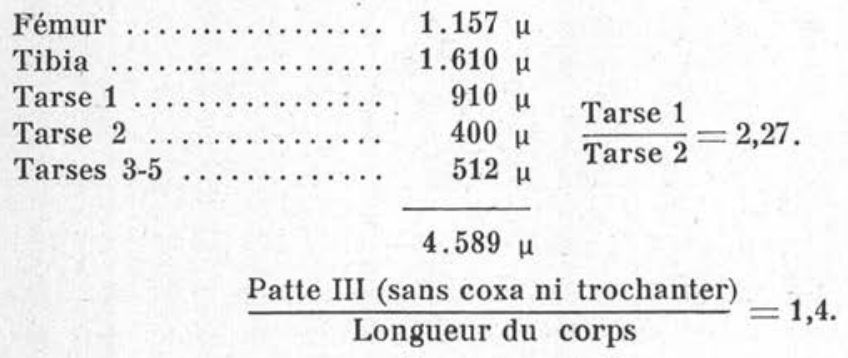

\section{Aile}

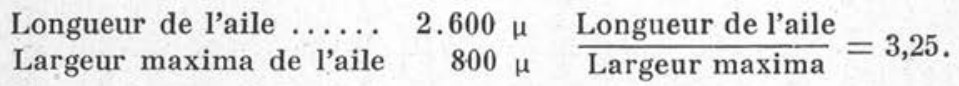

L'innervation est semblable à celle des ailes des mâles. Les deux grandes fourches sont sensiblement sur la même ligne. Le maximum de largeur est en arrière de $\mathrm{Cu}^{2}$.

Voici quelques rapports $\frac{\alpha}{\beta}$ et $\frac{\alpha}{\delta}$

$$
\frac{\alpha=495}{\beta=295}=167 \frac{\alpha=495}{\delta}=58,05 \frac{\alpha=610}{\beta=280}=2,17 \frac{\alpha=610}{\delta=100}=6,10
$$

$\alpha$ sensiblement égal à $\gamma$.

\section{Spermathèques}

Nous avons démonté une préparation, nous l'avons passée à la potasse et remontée ensuite dans le chloral-lacto-phénol afin de mieux voir les spermathèques. Elles ont la forme que nous avons représentée dans la figure 6 , donc elles sont tout à fait caractéristiques. 


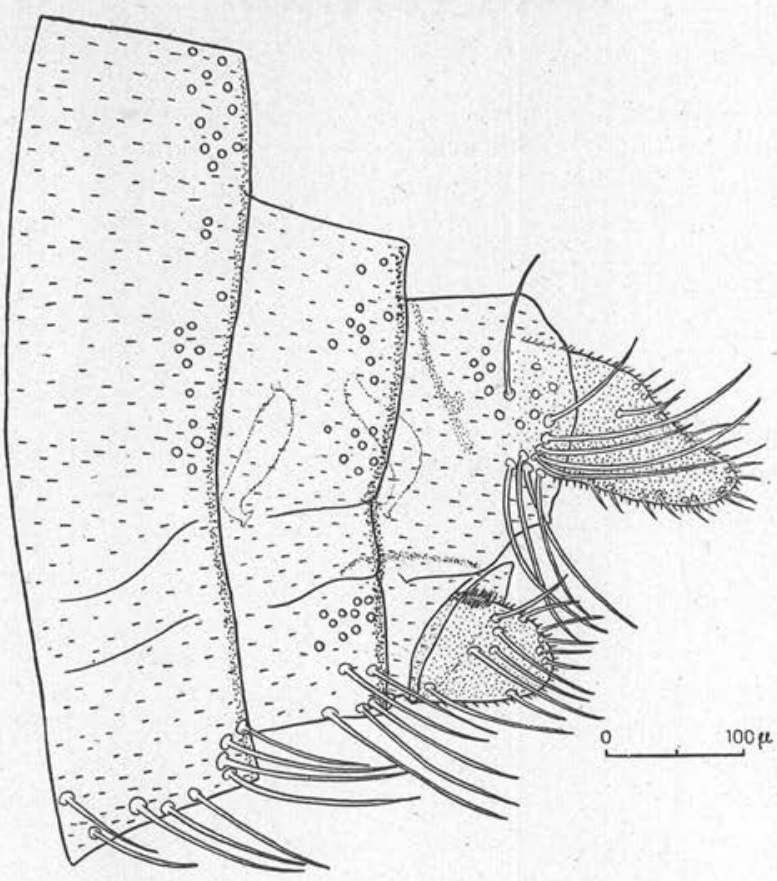

8

Fig. 5. - Phlebotomus chinensis. Partie terminale de l'abdomen d'une femelle. Les spermathèques sont représentés en pointillé.

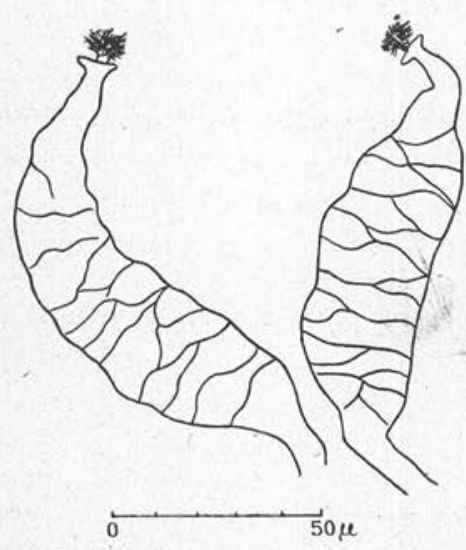

Fig. 6. - Phlebotomus chinensis. Spermathèques. 


\section{Dents pharyngiennes}

Le pharynx est très large $(100 \mu)$. Les dents, nombreuses, remontent sur une hauteur d'à peu près $75 \mu$. Il y a deux sortes de dents : celles de la partie supérieure sont très développées; elles sont encerclées à leur partie inférieure par un autre groupe de dents bien plus petites, mais à base d'implantation fort élargie, qui donnent par leur disposition

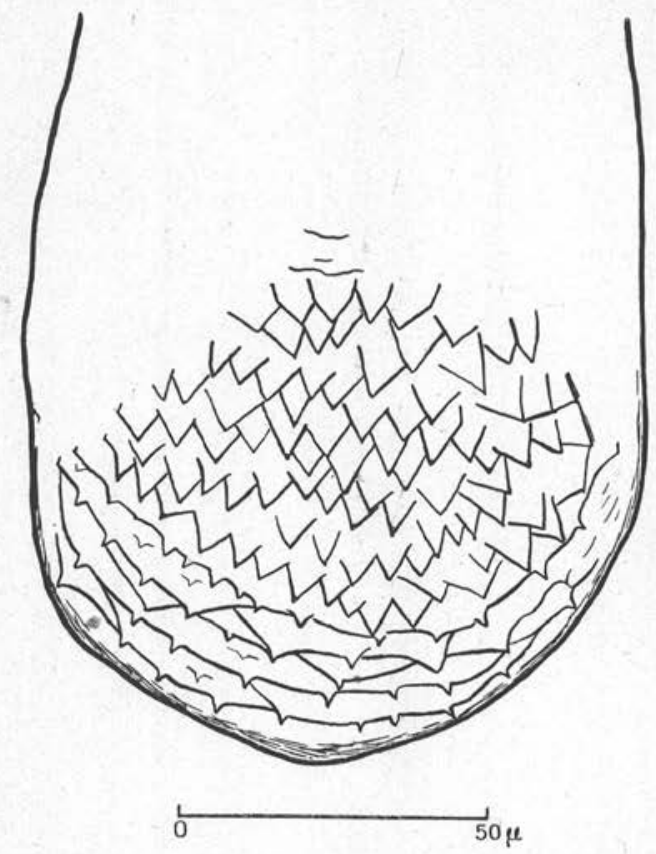

Fig. 7. - Phlebotomus chinensis. Armature pharyngienne de la femelle.

l'impression de lignes courbes parallèles (fig. 7). Nous devons remarquer que les dents supérieures sont moins colorées que dans les races européennes que nous avons étudiées, surtout celle de Bucarest. Il se pourrait que l'ancienneté des préparations ait provoqué cette décoloration.

\section{Pièces buccales}

Les pièces buccales, chitineuses, quoique difficiles à étudier et à examiner, pourront-elles nous être d'un certain secours dans les déterminations délicates? Perfiliew, dans ses études d'anatomie comparée des phlébotomes, nous montre une série de différences entre les pièces buccales des diverses espèces. Nous avons disséqué une des trois 
femelles afin de pouvoir observer attentivement ces pièces. La dissection nous a montré que le labre-épipharynx diffère du dessin que Perfiliew (1928) nous a donné de la même pièce pour le Phlebotomus major var. longiductus. Au lieu des 5 palettes terminales, on en a 6 dans le $P$. chinensis (fig. 8). Serait-ce une preuve que $P$. major var. longiductus décrit
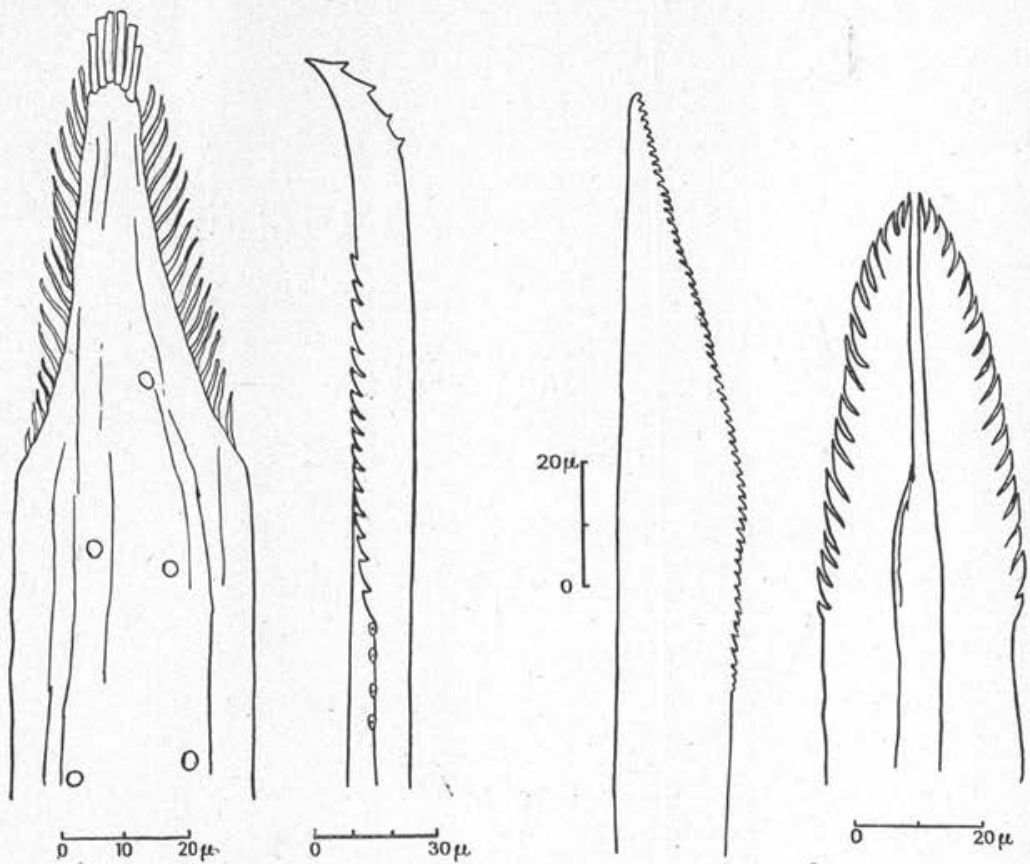

FIG. 8. - Phlebotomus chinensis. Epipharynx de la femelle, terminé par 6 palettes.

Fig. 9. - Phleboto-
mus chinensis. Maxille de la femelle.
Fig. 10. - Phleboto- Fig. 11. - Phlebomus chinensis. tomus chinensis. Mandibule de la Hypopharynx de femelle.

par Perfiliew et qui est à présent considéré comme synonyme de Phlebotomus chinensis n'est pas superposable à celui que nous décrivons ? Des recherches ultérieures avec des dissections plus nombreuses pourront trancher cette question.

Le maxille présente du côté externe de son extrémité apicale quatre fortes dents noirâtres (fig. 9). La rangée des petites dents internes arrive très près de l'extrémité antérieure. La mandibule (fig. 10) et l'hypopharynx (fig. 11), que nous représentons également, ne semblent avoir rien de bien caractéristique. 


\section{RÉSUMÉ}

Nous donnons la description détaillée d'une collection de Phlebotomus chinensis. Nous avons mesuré les divers organes et nous donnons une série de chiffres qui représentent les moyennes de ces mensurations.

La formule des tarses de Patton et Hindle, qui ne se retrouvait pas pour les formes européennes et qui nous embarrassait en conséquence lors de leur diagnose, n'existe également pas sur cette forme authentique de Chine. Elle doit donc être rayée de la diagnose de l'espèce. La largeur de l'aile est maxima en arrière de $\mathrm{Cu}^{2}$ pour les trois formes, de Chine, de Bucarest et de Skoplje.

Le rapport entre la longueur des filaments génitaux et celle de la pompe oscille entre 7 et 5,5. Sous ce rapport, les formes de Skoplje que nous avions identifiées au $P$. chinensis sont pareilles aux formes de la Chine.

$P$. chinensis de Bucarest, de même que $P$. major var. longiductus de Parrot, possède la pormule antennaire : $\frac{2}{\text { III-VII }}, \frac{1}{\text { VIII-XV }}$; tandis que chez $P$. chinensis de Chine, la formule antennaire est: 2

$\overline{\text { III }-\mathrm{XV}}$.

Parmi tous les organes que nous avons signalés, il n'y a que la formule antennaire et l'organe intromittent avec sa forme particulière et ses rapports de longueur avec les appendices intermédiaires qui semblent caractériser le mieux la race de Chine que nous avons étudiée.

Nous faisons suivre la description des phlébotomes femelles par celle de la dissection de l'appareil buccal, afin de chercher dans la conformation des pièces buccales d'autres caractères qui pourraient nous être utiles dans les déterminations. Nos résultats ne concordent pas avec ceux de Pefiliew pour les formes asiatiques.

Quant aux formes européennes nous y reviendrons prochainement.

\section{BiBLIOGRAPHIE}

AdLer et Theodor. - The distribution of sandflies and leishmaniasis in Palestina, Syria and Mesopotamia. Annals of tropical Medecine and Parasilology, XXIII, 1929, p. 269-306.

Magnitski et Gutzewitsch. - Zur Frage über die Veränderlichkeit einiger systematischer Merkmale der Arten der Gattung Phlebotomus. Centralblatt f. Bakt. und. Parasit,, Originale, XC, 1929, p. 199-208. 
Newstead (R.). - On the genus Phlebotomus. Part. III, Phlebotomus major, var. chinensis. Bull. of entomological research, VII, 1916, p. 191-192.

Nitzulescu (V.). - Contribution à l'étude des phlébotomes de Roumanie. Annales de Parasitologie, VII, 1929, p. 430-437.

- Sur quelques phlébotomes de Yougoslavie. Annales de Parasilologie, VII, 1929, p. 494-505.

Patron et Evans. - Insects, Tiks, Mites el venomous animals, Croydon, 1929.

Patton et Hindez. - Notes on the species of sandflies (genus Phlebotomus) of North China. Proceedings of the royal Society, B, C, 1926, p. 405-412.

Perfiliew (P.). - Zur vergleichende Anatomie von Phlebotomus. Zeitschrit für Parasitenkunde, I, 1928, p. 437-475.

Pinto (C.). - Phlebotomus neivai e Phlebotomus fischeri. Sobre o apparelho espicular dos phlebotomos e seu valor specifica. Sciencia medica, IV, 1926, p. $370-375$.

Sinton (J.). - The synonymy of the asialic species of Phlebotomus. Indian Journal of medical research, XVI, 1928, p. 297-324.

Laboratoire de parasitologie de la Faculté de médecine de Paris 